Bull. Korean Math. Soc. 51 (2014), No. 1, pp. 221-227

http://dx.doi.org/10.4134/BKMS.2014.51.1.221

\title{
A NOTE ON EXPONENTIAL ALMOST SURE STABILITY OF STOCHASTIC DIFFERENTIAL EQUATION
}

\author{
Xuerong Mao, Qingshuo Song, and Dichuan Yang
}

\begin{abstract}
Our goal is to relax a sufficient condition for the exponential almost sure stability of a certain class of stochastic differential equations. Compared to the existing theory, we prove the almost sure stability, replacing Lipschitz continuity and linear growth conditions by the existence of a strong solution of the underlying stochastic differential equation. This result is extendable for the regime-switching system. An explicit example is provided for the illustration purpose.
\end{abstract}

\section{Introduction}

Let $\left(\Omega, \mathcal{F}, \mathbb{P},\left\{\mathcal{F}_{t}\right\}\right)$ be a filtered probability space satisfying general conditions, on which $B(t) \in \mathbb{R}^{m}$ is a standard $m$-dimensional Brownian motion. Consider a stochastic differential equation (SDE) of

$$
d X(t)=f(X(t), t) d t+g(X(t), t) d B(t), X\left(t_{0}\right)=x_{0},
$$

where $X(t) \in \mathbb{R}^{d}, f: \mathbb{R}^{d} \times \mathbb{R}_{+} \rightarrow \mathbb{R}^{d}, g: \mathbb{R}^{d} \times \mathbb{R}_{+} \rightarrow \mathbb{R}^{d \times m}$. It is well known that SDE (1) has a unique strong solution under the following assumption:

Assumption 1. $f$ and $g$ obey the local Lipschitz continuity and Linear growth condition.

In the past a few decades, the stability of such a system has been well studied as an important feature, see more details in $[1,3]$ and the references therein. Our interest of this note is to relax a sufficient condition for the almost sure exponential stability based on the existing result of Theorem 3.3 of the book [3].

For convenience, we define an operator $L$ by, for all $\varphi \in C^{2,1}\left(\mathbb{R}^{d} \times \mathbb{R}_{+} ; \mathbb{R}\right)$,

$$
L \varphi(x, t)=\varphi_{t}(x, t)+\varphi_{x}(x, t) \cdot f(x, t)+\frac{1}{2} \operatorname{Trace}\left(\varphi_{x x}(x, t)\left(g g^{T}\right)(x, t)\right) .
$$

Received February 19, 2013; Revised June 28, 2013.

2010 Mathematics Subject Classification. 60G52, 60G17.

Key words and phrases. almost sure stability, stochastic differential equation, regimeswitching, Besel squared process.

The research is partially supported in part by CityU Strategic Research Grant No. 7002818 and Research Grants Council of Hong Kong No. CityU-109613. 
Theorem 3.3 of the book [3] provides a sufficient condition for the exponential almost sure stability under the following assumptions, and its proof is referred to $[3]$.

Theorem 1 (Theorem 3.3 of [3]). Suppose SDE (1) satisfies Assumption 1 and

$$
f(0, t)=g(0, t)=0, \forall t \in\left[t_{0}, \infty\right) .
$$

Also, assume that there exist a function $V \in C^{2,1}\left(\mathbb{R}^{d} \times\left[t_{0}, \infty\right) ; \mathbb{R}_{+}\right)$, and constants $p>0, c_{1}>0, c_{2} \in \mathbb{R}, c_{3} \geq 0$ such that for all $x \neq 0$ and $t \geq t_{0}$,

(H1) $c_{1}|x|^{p} \leq V(x, t)$,

(H2) $L V(x, t) \leq c_{2} V(x, t)$,

(H3) $\left|V_{x}(x, t) \cdot g(x, t)\right|^{2} \geq c_{3} V^{2}(x, t)$.

Then,

$$
\limsup _{t \rightarrow \infty} \frac{1}{t} \log \left|X\left(t ; t_{0}, x_{0}\right)\right| \leq-\frac{c_{3}-2 c_{2}}{2 p} \quad \text { a.s. }
$$

In particular, SDE (1) is exponentially stable in almost sure sense, if $c_{3}>2 c_{2}$.

Together with Assumption 1, equation (2) implies zero is an absorbing state for $X(t)$. Moreover, one can also conclude $X(t)>0$ almost surely if the initial $x_{0}$ is given strictly positive.

In this below, we will present a stability result with weaker sufficient condition. In particular, we will remove Assumption 1 and (2). Instead, we impose the existence of the strong solution as follows.

Assumption 2. SDE (1) has a strong solution for any initial $x_{0}$.

The proof of the next result is relegated to the next section for better illustration.

Theorem 2 (Main stability result). If SDE (1) satisfies Assumption 2, and assume that there exist a function $V \in C^{2,1}\left(\mathbb{R}^{d} \times\left[t_{0}, \infty\right) ; \mathbb{R}_{+}\right)$, and constants $p>0, c_{1}>0, c_{2} \in \mathbb{R}, c_{3} \geq 0$ such that for $t \geq t_{0}$, (H1)-(H3) are satisfied. Then

$$
\limsup _{t \rightarrow \infty} \frac{1}{t} \log \left|X\left(t ; t_{0}, x_{0}\right)\right| \leq-\frac{c_{3}-2 c_{2}}{2 p} \quad \text { a.s. }
$$

In the above, we extend the definition to $\log 0=-\infty$.

It is well known that Assumption 1 is only a sufficient condition for the existence of a strong solution, but not a necessary condition (see more discussions in Chapter 5 of [2]). In the next section after the proof of Theorem 2, we will provide an example, which can be verified to be almost surely exponential stable by Theorem 2, but not by Theorem 1. Section 3 includes an extension of the result to regime-switching diffusion and other possible generalizations.

\section{Proof of the main result and an example}

In this section, we will provide a proof of Theorem 2 and an example for the illustration purpose. 


\subsection{Proof of Theorem 2}

In the above Assumption 2 we do not exclude possibly zero solutions. Therefore, although $\log V(x(t), t)$ is well defined for all $t \in(0, \infty)$ under extended $\log$ arithm, it is not directly applicable to Ito's formula to calculate $d \log V(x(t), t)$ due to possible value $\log 0$. For this reason, we define a new strictly positive function

$$
V^{\eta}(x, t)=V(x, t)+e^{-\eta t}
$$

for some constant $\eta>0$ to be given by (9). By Ito's formula on $V^{\eta}$, we obtain

$$
d V^{\eta}(X(t), t)=\left(L V(X(t), t)-\eta e^{-\eta t}\right) d t+\left(V_{x} \cdot g\right)(X(t), t) d B_{t} .
$$

For the strictly positive function $V^{\eta}$, we can apply Ito's formula on $\log V^{\eta}(X(t)$, t) to obtain

$$
\begin{aligned}
& d \log V^{\eta}(X(t), t) \\
= & \frac{1}{V^{\eta}(X(t), t)} d V^{\eta}(X(t), t)-\frac{1}{2} \frac{1}{V^{\eta}(X(t), t)^{2}} d\left\langle V^{\eta}, V^{\eta}\right\rangle(X(t), t) \\
= & \left(\frac{L V}{V^{\eta}}(X(t), t)-\frac{1}{2}\left|\frac{V_{x} \cdot g}{V^{\eta}}\right|^{2}(X(t), t)-\frac{\eta e^{-\eta t}}{V^{\eta}}(X(t), t)\right) d t \\
& +\frac{V_{x} \cdot g}{V^{\eta}}(X(t), t) d B_{t} .
\end{aligned}
$$

Rewrite it into integral form

$$
\begin{aligned}
& \log V^{\eta}(X(t), t) \\
= & \log V^{\eta}\left(x_{0}, t_{0}\right) \\
& +\int_{t_{0}}^{t}\left(\frac{L V}{V^{\eta}}(X(s), s)-\frac{1}{2}\left|\frac{V_{x} \cdot g}{V^{\eta}}\right|^{2}(X(s), s)-\frac{\eta e^{-\eta s}}{V^{\eta}}(X(s), s)\right) d s \\
& +\int_{t_{0}}^{t} \frac{V_{x} \cdot g}{V^{\eta}}(X(s), s) d B_{s} .
\end{aligned}
$$

Denote the last term by

$$
M(t)=\int_{t_{0}}^{t} \frac{V_{x} \cdot g}{V^{\eta}}(X(s), s) d B_{s} .
$$

Note that $M(t)$ is a continuous martingale with initial value $M\left(t_{0}\right)=0$. Assign $\epsilon \in(0,1)$, by exponential martingale inequality (see $[5,7]$ )

$$
\mathbb{P}\left(\sup _{0 \leq t \leq n}\left[M(t)-\frac{\epsilon}{2} \int_{t_{0}}^{t} \frac{\left|V_{x} \cdot g\right|^{2}}{\left|V^{\eta}\right|^{2}}(X(s), s) d s\right]>\frac{2}{\epsilon} \log n\right) \leq \frac{1}{n^{2}} .
$$

Applying Borel-Cantelli lemma, we will see that for almost all $\omega \in \Omega$, there is an integer $n_{0}=n_{0}(\omega)$ such that when $n \geq n_{0}$

$$
M(t) \leq \frac{2}{\epsilon} \log n+\frac{\epsilon}{2} \int_{t_{0}}^{t} \frac{\left|V_{x} \cdot g\right|^{2}}{\left|V^{\eta}\right|^{2}}(X(s), s) d s
$$

for all $0 \leq t \leq n$. 
Using (H2) and (H3) on (5) and (6), it leads to the inequality

$$
\log V^{\eta}(X(t), t) \leq \log V^{\eta}\left(x_{0}, t_{0}\right)+\int_{t_{0}}^{t} I(s) d s+\frac{2}{\epsilon} \log n
$$

where

$$
I(s)=\frac{c_{2} V}{V^{\eta}}(X(s), s)-\frac{\frac{1}{2}(1-\epsilon) c_{3}|V|^{2}}{\left|V^{\eta}\right|^{2}}(X(s), s)-\frac{\eta e^{-\eta s}}{V^{\eta}}(X(s), s) .
$$

Define $G: \mathbb{R}_{+} \rightarrow \mathbb{R}$ by

$$
G(y)=\frac{c_{2} y}{y+1}-\frac{(1-\epsilon) c_{3} y^{2}}{2(y+1)^{2}}-\frac{\eta}{y+1} .
$$

In terms of function $G(\cdot), I(s)$ has representation of

$$
I(s)=G\left(V(X(s), s) \cdot e^{\eta s}\right) .
$$

Now, by taking special $\eta$ of

$$
\eta:=(1-\epsilon) c_{3}-c_{2}
$$

one can observe that

$$
G^{\prime}(y)=\frac{(1-\epsilon) c_{3}}{(y+1)^{3}} \geq 0, \forall y \in(0, \infty)
$$

which means $G(\cdot)$ is an non-decreasing function on $(0, \infty)$. Hence, we can find an upper bound of $G$ by calculating its limit at infinity, that is

$$
G(y) \leq c_{2}-\frac{1}{2}(1-\epsilon) c_{3}=\lim _{y \rightarrow \infty} G(y), \forall y \in \mathbb{R}_{+}
$$

Together with (8), we have

$$
I(s) \leq c_{2}-\frac{1}{2}(1-\epsilon) c_{3} .
$$

With this inequality, we rewrite (7) as

(10) $\log V^{\eta}(X(t), t) \leq \log V^{\eta}\left(x_{0}, t_{0}\right)+\left(c_{2}-\frac{1}{2}(1-\epsilon) c_{3}\right)\left(t-t_{0}\right)+\frac{2}{\epsilon} \log n$ for all $0 \leq t \leq n$. Therefore, if $t$ is chosen as $n-1 \leq t \leq n$, then $\frac{1}{t} \log V^{\eta}(X(t), t) \leq \frac{1}{t} \log V^{\eta}\left(x_{0}, t_{0}\right)+\left(c_{2}-\frac{1}{2}(1-\epsilon) c_{3}\right) \frac{t-t_{0}}{t}+\frac{2}{\epsilon} \frac{\log (t+1)}{t}$.

Passing the limit $t \rightarrow \infty$, we obtain

$$
\limsup _{t \rightarrow \infty} \frac{1}{t} \log V^{\eta}(X(t), t) \leq\left(c_{2}-\frac{1}{2}(1-\epsilon) c_{3}\right) .
$$

Finally, we reach the desired result (3) as a consequence of the arbitrariness of $\epsilon \in(0,1),(\mathrm{H} 1)$, and $V \leq V^{\eta}$. 


\subsection{Example}

Consider SDE given by

$$
d X(t)=-X(t) d t+2 e^{-t / 2} X^{1 / 2}(t) d B(t), \quad X(0) \geq 0 .
$$

SDE (11) violates Assumption 1 due to its coefficient of $d B(t)$. On the other hand, SDE (11) satisfies Assumption 2, since we can find a strong solution of the form $X(t)=e^{-t} Y(t)$, where $Y(t)$ is a squared Bessel process with dimension $\delta=0$, namely $B E S Q^{0}$, which satisfies

$$
d Y(t)=2 \sqrt{Y(t)} d B(t), Y_{0}=x_{0}
$$

(see Chapter XI of [6]). In fact, the above $X(t)$ is the unique solution of SDE (11) by Theorem IX.3.5 of [6].

Set Liapunov function $V(x)$ by $V(x)=x$ on $(x, t) \in \mathcal{O}:=\mathbb{R}_{+} \times \mathbb{R}_{+}$. We only define $V$ on $\mathcal{O}$, since $X(t)>0$ almost surely provided that $x_{0}>0$. Then, we can verify that $V$ satisfies (H1)-(H3) with the constants $p=1, c_{1}=1, c_{2}=$ $-1, c_{3}=0$. Thus, $X(t)$ is exponentially stable in the almost sure sense, with

$$
\limsup _{t \rightarrow \infty} \frac{1}{t} \log |X(t)| \leq-1 \text {. }
$$

\section{Further extensions}

In this part, we will present two generalizations of the stability result. The proof can be completed using exactly the same methodologies discussed above.

\subsection{Regime-switching model}

Let $\gamma(\cdot)$ be a continuous Markov chain taking value in a finite state space $\mathcal{M}=\{1,2, \ldots, m\}$ with generator $Q(x)=q_{i j}(x)$ satisfying $\left(q_{i j}(x)\right)_{i, j \in \mathcal{M}} \geq 0$ for $j \neq i$ and $\sum_{j \in \mathcal{M}} q_{i j}(x)=0$ for all $x \in \mathbb{R}^{d}$ and $i \in \mathcal{M}$. Associated to the Markov chain $\gamma$, we consider following stochastic differential equation:

(12) $d X(t)=f(X(t), \gamma(t), t) d t+g(X(t), \gamma(t), t) d B(t), X\left(t_{0}\right)=x_{0}, \gamma(t)=\gamma_{0}$.

The stochastic differential equation of the form (12) is often referred to regimeswitching diffusion. The applications of such a system have been witnessed in various areas, such as signal processing, production planning, biological systems (see more in $[4,8,9])$. The generator of $(12)$ can be written by, for $\varphi \in$ $C^{2,1}\left(\mathbb{R}^{d} \times \mathcal{M} \times \mathbb{R}_{+} ; \mathbb{R}\right)$

$$
\begin{aligned}
L \varphi(x, i, t)= & \varphi_{t}(x, i, t)+\varphi_{x}(x, i, t) \cdot f(x, i, t) \\
& +\frac{1}{2} \operatorname{Trace}\left(\varphi_{x x}(x, i, t)\left(g g^{T}\right)(x, i, t)\right) \\
& +\sum_{\mathcal{M} \ni j \neq i} q_{i j}(x)(g(x, j, t)-g(x, i, t)) .
\end{aligned}
$$

If we go through similar steps in Section 2, we obtain the following result on the stability, and the proof is omitted to keep this note brief. 
Theorem 3. Suppose SDE (12) has the unique strong solution, and there exist a function $V \in C^{2,1}\left(\mathbb{R}^{d} \times \mathcal{M} \times\left[t_{0}, \infty\right) ; \mathbb{R}_{+}\right)$, and constants $p>0, c_{1}>0, c_{2} \in$ $\mathbb{R}, c_{3} \geq 0$ such that for all $x \neq 0, i \in \mathcal{M}$ and $t \geq t_{0}$,

(H1') $c_{1}|x|^{p} \leq V(x, i, t)$,

(H2') $L V(x, i, t) \leq c_{2} V(x, i, t)$,

(H3') $\left|V_{x}(x, i, t) \cdot g(x, i, t)\right|^{2} \geq c_{3} V^{2}(x, i, t)$.

Then,

$$
\limsup _{t \rightarrow \infty} \frac{1}{t} \log |X(t)| \leq-\frac{c_{3}-2 c_{2}}{2 p} \quad \text { a.s. }
$$

In particular, SDE (12) is exponentially stable in almost sure sense, if $c_{3}>2 c_{2}$.

\subsection{Delay systems}

In this part, we consider stochastic differential delay equations (see [10] and the references therein):

$$
d X(t)=f(X(t), X(t-\delta(t)), t) d t+g(X(t), X(t-\delta(t)), t) d B(t)
$$

with initial data

$$
X(t)=\psi(t), \quad \forall t \in\left[t_{0}-\delta\left(t_{0}\right), t_{0}\right],
$$

where $X(t) \in \mathbb{R}^{d}, f: \mathbb{R}^{d} \times \mathbb{R}^{d} \times \mathbb{R}_{+} \rightarrow \mathbb{R}^{d}, g: \mathbb{R}^{d} \times \mathbb{R}^{d} \times \mathbb{R}_{+} \rightarrow \mathbb{R}^{d \times m}$. We define an operator $L: C^{2,1}\left(\mathbb{R}^{d} \times \mathbb{R}_{+} ; \mathbb{R}\right) \mapsto L^{0}\left(\mathbb{R}^{d} \times \mathbb{R}^{d} \times \mathbb{R} ; \mathbb{R}\right)$ by, for all $\varphi \in C^{2,1}\left(\mathbb{R}^{d} \times \mathbb{R}_{+} ; \mathbb{R}\right)$,

$$
L \varphi(x, y, t)=\varphi_{t}(x, t)+\varphi_{x}(x, t) \cdot f(x, y, t)+\frac{1}{2} \operatorname{Trace}\left(\varphi_{x x}(x, t)\left(g g^{T}\right)(x, y, t)\right) .
$$

Assumption 3. SDE (13) has a strong solution for any initial $x$.

Theorem 4. If SDE (13) satisfies Assumption 3 and

$$
f(0, y, t)=g(0, y, t)=0, \forall(y, t) \in \mathbb{R}^{d} \times \mathbb{R}_{+},
$$

and assume that there exist a function $V \in C^{2,1}\left(\mathbb{R}^{d} \times \mathbb{R}_{+} ; \mathbb{R}_{+}\right)$, and constants $p>0, c_{1}>0, c_{2} \in \mathbb{R}, c_{3} \geq 0$ such that for $t \geq t_{0}$,

(H1") $c_{1}|x|^{p} \leq V(x, t)$,

(H2") $L V(x, y, t) \leq c_{2} V(x, t)$,

(H3") $\left|V_{x}(x, t) g(x, y, t)\right|^{2} \geq c_{3} V^{2}(x, t)$.

Then

$$
\limsup _{t \rightarrow \infty} \frac{1}{t} \log \left|x\left(t ; t_{0}, x_{0}\right)\right| \leq-\frac{c_{3}-2 c_{2}}{2 p} \quad \text { a.s. }
$$

\section{References}

[1] R. Z. Has'minskiı̌, Stochastic Stability of Differential Equations, Volume 7 of Monographs and Textbooks on Mechanics of Solids and Fluids: Mechanics and Analysis, Sijthoff \& Noordhoff, Alphen aan den Rijn, 1980.

[2] I. Karatzas and S. E. Shreve, Methods of Mathematical Finance, Volume 39 of Applications of Mathematics (New York), Springer-Verlag, New York, 1998. 
[3] X. Mao, Stochastic Differential Equations and Their Applications, Horwood Publishing Series in Mathematics \& Applications. Horwood Publishing Limited, Chichester, 1997.

[4] X. Mao and C. Yuan, Stochastic Differential Equations with Markovian Switching, Imperial College Press, London, 2006

[5] M. Métivier, Semimartingales, Volume 2 of de Gruyter Studies in Mathematics, Walter de Gruyter \& Co., Berlin, 1982.

[6] D. Revuz and M. Yor, Continuous Martingales And Brownian Motion, Third edition, Springer-Verlag, Berlin, 1999.

[7] J. A. Yan, Yang yu suiji jifen yinlun, Shanghai Science and Technology Publishing House, Shanghai, 1981.

[8] G. G. Yin and Q. Zhang, Continuous-time Markov chains and applications, Volume 37 of Applications of Mathematics (New York), Springer-Verlag, New York, 1998.

[9] G. G. Yin and C. Zhu, Hybrid switching diffusions, Properties and applications, Volume 63 of Applications of Mathematics (New York), Springer-Verlag, New York, 2010.

[10] C. Yuan and X. Mao, Robust stability and controllability of stochastic differential delay equations with Markovian switching, Automatica J. IFAC 40 (2004), no. 3, 343-354.

Xuerong MaO

Department of Mathematics and Statistics

UNIVERSITY OF STRATHCLYDE

$\mathrm{UK}$

E-mail address: x.mao@strath.ac.uk

QINGSHUO SONG

Department of Mathematics

City University of Hong Kong

HONG KONG

E-mail address: qingsong@cityu.edu.hk

DiCHUAN YANG

Department of Mathematics

City University of Hong Kong

Hong Kong

E-mail address: dyang5@student.cityu.edu.hk 